\title{
The human gut archaeome: identification of diverse haloarchaea in Korean subjects
}

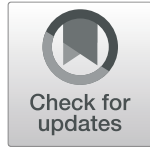

\author{
Joon Yong Kim" ${ }^{1 \dagger}$, Tae Woong Whon ${ }^{1 \dagger}$, Mi Young Lim², Yeon Bee Kim, Namhee Kim, Min-Sung Kwon', \\ Juseok Kim', Se Hee Lee ${ }^{1}$, Hak-Jong Choi', In-Hyun Nam³ , Won-Hyong Chung ${ }^{2}$, Jung-Ha Kim, Jin-Woo Bae', \\ Seong Woon Roh ${ }^{1 *}$ (D) and Young-Do Nam ${ }^{2^{*}}$
}

\begin{abstract}
Background: Archaea are one of the least-studied members of the gut-dwelling autochthonous microbiota. Few studies have reported the dominance of methanogens in the archaeal microbiome (archaeome) of the human gut, although limited information regarding the diversity and abundance of other archaeal phylotypes is available.

Results: We surveyed the archaeome of faecal samples collected from 897 East Asian subjects living in South Korea. In total, $42.47 \%$ faecal samples were positive for archaeal colonisation; these were subsequently subjected to archaeal $16 \mathrm{~S}$ rRNA gene deep sequencing and real-time quantitative polymerase chain reaction-based abundance estimation. The mean archaeal relative abundance was $10.24 \pm 4.58 \%$ of the total bacterial and archaeal abundance. We observed extensive colonisation of haloarchaea (95.54\%) in the archaea-positive faecal samples, with 9.63\% mean relative abundance in archaeal communities. Haloarchaea were relatively more abundant than methanogens in some samples. The presence of haloarchaea was also verified by fluorescence in situ hybridisation analysis. Owing to large inter-individual variations, we categorised the human gut archaeome into four archaeal enterotypes. Conclusions: The study demonstrated that the human gut archaeome is indigenous, responsive, and functional, expanding our understanding of the archaeal signature in the gut of human individuals.
\end{abstract}

Keywords: Human gut, Population-level metataxonomic analysis, Archaeome, Haloarchaea, Archaeal enterotype

\section{Background}

The human gut harbours various biological entities such as bacteria, archaea, unicellular eukaryotes, and viruses [1]. These microbial entities constitutively contribute to the microbial signature, thereby maintaining the inherent characteristics of the gastrointestinal tract. To date, studies have focused on the genetic and functional traits of gut bacteria [2]. The introduction of next-generation sequencing technologies in gut microbiology has revealed

\footnotetext{
*Correspondence: swroh@wikim.re.kr; youngdo98@kfri.re.kr

†Joon Yong Kim and Tae Woong Whon contributed equally to this work. ${ }^{1}$ Microbiology and Functionality Research Group, World Institute of Kimchi, Gwangju 61755, Republic of Korea

${ }^{2}$ Research Group of Healthcare, Research Division of Food Functionality, Korea Food Research Institute, Jeollabuk-do 55365, Republic of Korea Full list of author information is available at the end of the article
}

the identity of gut microorganisms. Metagenomics-based gut microbial surveys on healthy populations [3, 4], as well as on those with various illnesses [5], have shed light on the characteristics of the bacterial microbiome under both eubiotic and dysbiotic conditions. The rigid basic information regarding the natural members of the bacterial microbiota has prompted further studies on the function of these microorganisms.

The domain Archaea was proposed as a separate group of prokaryotes in 1990 based on the ribosomal RNA gene sequences. Although most archaea are thought to be extremophiles, living in harsh environments, mesophilic archaea have been identified in moderate environments, such as the soil and ocean [6,7]. Additionally, several studies have confirmed the presence of archaea on the 
human skin [8] and in the mouth [9] and gut [10, 11]. Although they are relatively minor, archaea are an important component of the human microbiome [12], and thus might have a complex community composition and structure on various human body sites.

Information regarding the community-based genetic and functional traits of archaea in animal habitats is scarce. We have previously reported occurrences of diverse members of extremely halophilic archaea (haloarchaea) in avian plumage [13], as well as in food samples such as salt-fermented seafood [14] and solar salts $[15,16]$. In the human gut where the microbial entities thrive more abundantly than in other parts of the human body, the archaeome consisted mostly of methane-producing archaea (methanogens), of which, members belonging to the orders Methanobacteriales (including Methanobrevibacter smithii and Methanosphaera stadtmanae) and Methanomassiliicoccales (including Methanomethylophilaceae) are predominant [17]. However, studies using culture-independent approaches have reported that not all archaea in the human gut are methanogens. For instance, our previous study was the first to report the presence of haloarchaea in faecal samples of Korean subjects in 2008 based on a conventional molecular ecology method [18]. More recently, viable haloarchaeal strains (belonging to the genus Haloferax) were isolated from human faeces [19, 20], and two genome sequences of the human gutderived haloarchaeal strains (i.e. Haloferax massiliensis and Halorubrum lipolyticum) are currently available [21]. However, the presence of the haloarchaeal community and their collective genomes (hereafter termed 'haloarchaeome') in the human gut was not confirmed despite the use of metataxonomic analysis, i.e. the archaeal 16S rRNA gene-targeted amplicon sequencing. In addition, archaeal members from the orders Sulfolobales and Nitrososphaerales have been detected in the human gut [17]. These observations suggest that the diversity and/or abundance of the human gut archaeome may vary with host factors, including diet and age. Methodological pitfalls (such as the selection of primer pairs and the sequence processing pipeline used) may also contribute to the low resolution of the human gut archaeome [22]. Collectively, there is no sufficient information on the identity of archaea in the gut environment.

In this study, we conducted a population-level metataxonomic analysis of the human gut archaeome. First, we screened 897 faecal samples collected from a cohort of Koreans, of which, 381 archaea-positive faecal samples were subjected to deep sequencing of the 16S rRNA gene amplicons and real-time quantitative polymerase chain reaction (PCR)-based abundance estimation, as well as Fluorescence in situ hybridisation (FISH) analysis. The Korean gut archaeome featured large inter- individual variation. We categorised the human gut archaeome into four archaeal enterotypes, i.e. the Methanobacteriaceae-, Methanomethylophilaceae-, Halo feracaceae- and the unclassified Euryarchaeota-dominated archaeome. We further assessed the correlation between the host metadata (dietary nutrients, food categories and clinical phenotypes) and the abundances of archaeal taxa to evaluate the host factors affecting the community structure of the human gut archaeome. Overall, we have attempted to understand the archaeal signature in the human gut.

\section{Results}

\section{Extensive profiling of the Korean gut archaeome}

This study includes faecal samples collected from 897 East Asian subjects living in South Korea. Although the detection of archaea is highly dependent on the methodology used, a maximum prevalence of $23 \%$ and $97.5 \%$ has been reported for the methanogens belonging to the order Methanobacteriales: Methanobrevibacter smithii and Methanosphaera stadtmanae, respectively, in the human gastrointestinal tract [12]. We first determined the presence of archaeal colonisation in all samples using an archaea-specific primer set. The results showed that 381 out of 897 faecal samples (42.47\%) were positive for archaeal colonisation, and the positive samples were subsequently subjected to deep sequencing of the gut archaeome. In total, 275,909,328 reads were obtained from the Illumina Hiseq ${ }^{\mathrm{TM}} \mathrm{X}$ platform. After quality control, the remaining 193,370,457 reads (mean: 507,534 reads per sample; median: 240,156) were subjected to further analysis. The rarefaction analysis based on observed amplicon sequence variants (ASVs) showed that the sequencing depth had reached saturation (see Additional file 1: Supplementary Fig. S1a). Annotation of the archaeal 16S rRNA gene sequences to the SILVA database led to the prediction of 685 ASVs in the Korean gut archaeome (see Additional file 1: Supplementary Fig. S2).

The taxonomic classification of the gut archaeome revealed the predominance of sequences assigned to the phylum Euryarchaeota, followed by the phylum Crenarchaeota (Fig. 1a, b). At the genus level, the Korean gut archaeome showed proportionally abundant sequences assigned to the methanogen group; the genera Methanobrevibacter and Methanosphaera in the family Methanobacteriaceae of the order Methanobacteriales were mostly proportionally abundant $(54.89 \%$ and $25.68 \%$ relative abundance, respectively) with minor contributions from the unclassified Methanomethylophilaceae. In particular, the Korean gut archaeome contained haloarchaea-assigned sequences with $9.63 \%$ mean relative abundance; sequences belonging to the genera Halolamina, Haloplanus, Halorubrum, Halobacterium, 


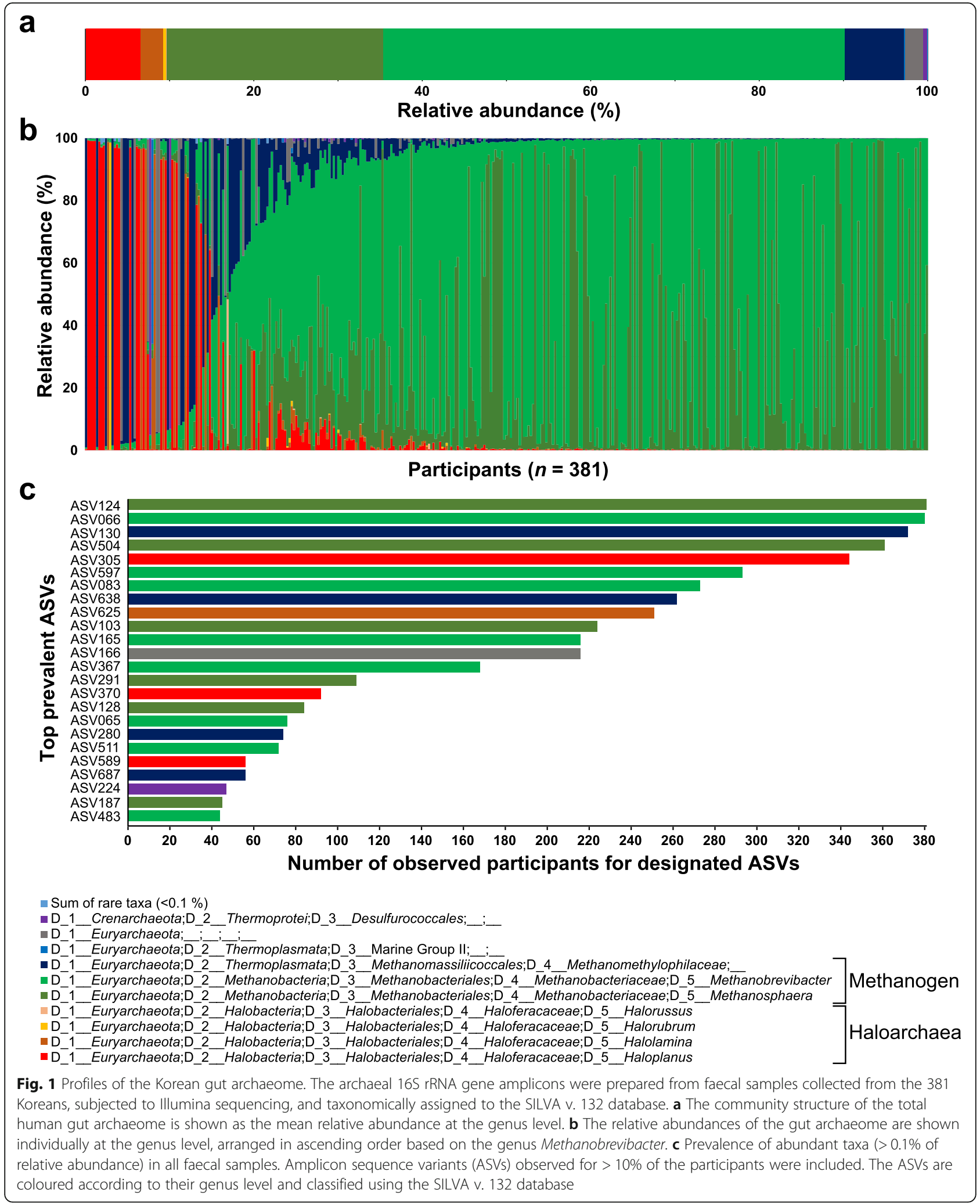

Haloterrigena, Natronomonas, Halarchaeum, Haloarcula, Halonotius and Halorussus were also detected. At the individual level, certain participants harboured the haloarchaea-dominated archaeal community structure (i.e. haloarchaea-assigned sequences showed 99.33\% relative abundance; Fig. 1b). Next, we assessed the core 
ASVs, i.e. ASVs detected extensively in all faecal samples. The methanogen-assigned ASVs (e.g. ASV124, ASV066 and ASV130) were detected in $>97 \%$ of the total samples. In contrast, haloarchaea-assigned ASVs (e.g. ASV305 assigned to the genus Haloplanus) were detected in $95.54 \%$ of the archaea-positive samples (Fig. 1c), suggesting that members belonging to both methanogens and haloarchaea might be the soft core microbial component, i.e. over $95 \%$ detection rate but not $100 \%$.

\section{Abundance estimation of the human gut archaea}

The abundances of bacterial cells $\left(10^{9}\right.$ to $10^{11}$ bacteria $\mathrm{g}^{-1}$ faeces) in the human gut have been well described $[23,24]$. We attempted to estimate the archaeal abundance by determining the archaea/(archaea + bacteria) ratios. We randomly selected 150 samples from the 381 archaeome-positive faecal samples and quantified both the archaeal and bacterial 16S rRNA gene copy numbers using real-time quantitative PCR. The results showed that the archaeal abundance was $10.24 \pm 4.58 \%$ (mean \pm standard deviation, SD) of the total bacterial and archaeal abundance (Fig. 2). The currently available information suggests that archaea and bacteria possess a mean of 1.7 and 5.0 16S rRNA genes per genome, respectively (https://rrndb.umms.med.umich.edu/ [25]). Based on this, we attempted to correct the gut archaeal abundance by the number of $16 \mathrm{~S}$ rRNA genes. As shown in Fig. 2, the adjusted archaeal abundance accounted for $22.35 \pm 7.90 \%$ (mean \pm SD) of the total bacterial and archaeal abundance.

\section{Compositional characterisation of the Korean gut archaeome}

One of the major features of the human bacterial microbiome is inter-individual variation commonly observed even in healthy individuals [26]. Our metataxonomic analysis revealed remarkable differences in the archaeal structure of individual Korean guts (Fig. 1). Therefore, we assessed the compositional features of the gut archaeome of 342 subsampled individuals (i.e. the number of sequences was evenly normalised at a sampling depth of 10,000 across the subjects; see Additional file 1: Supplementary Fig. S1b). Principal coordinate analysis (PCoA) of the weighted UniFrac distance matrix

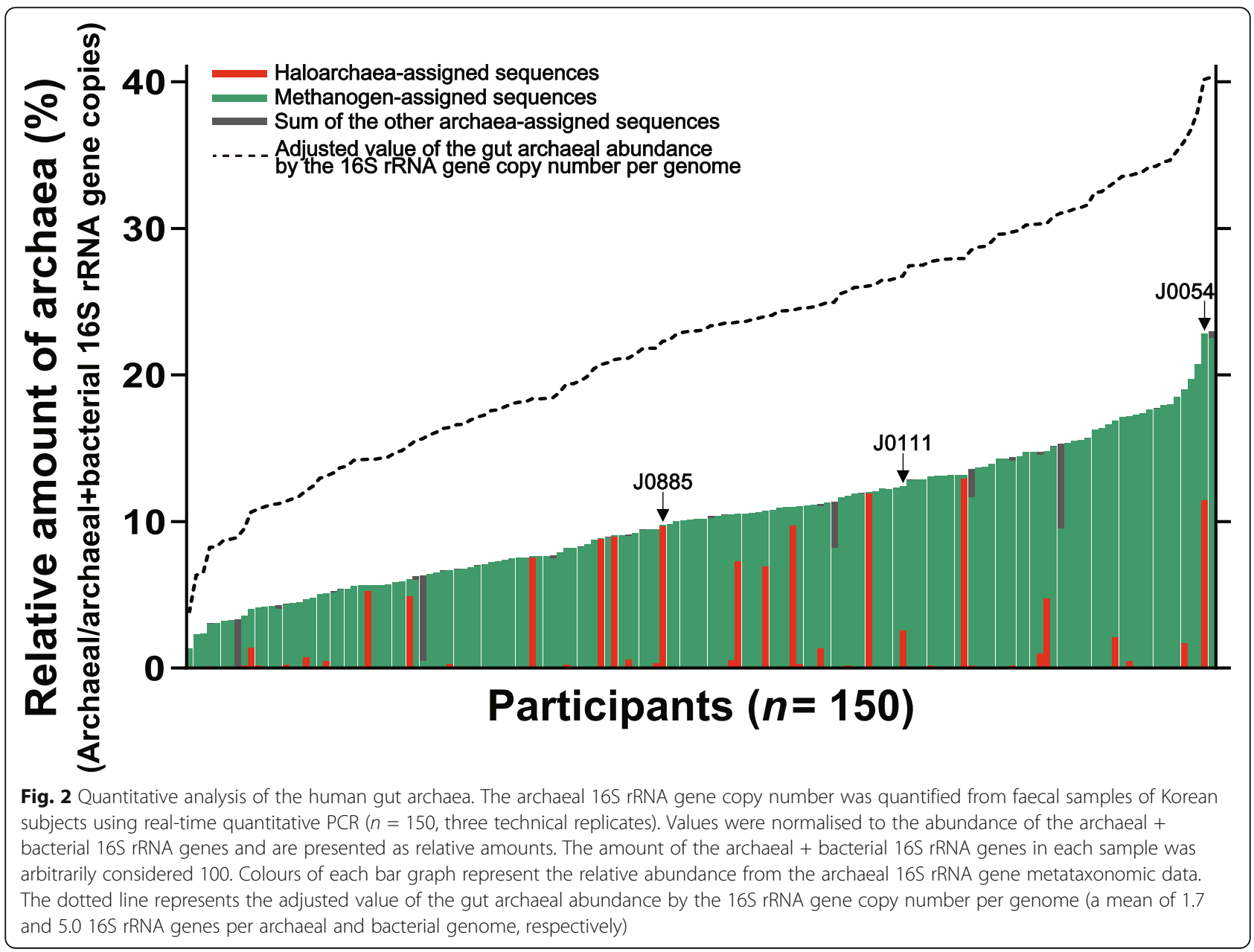


revealed that the per cent relative abundances of several key archaeal taxa, i.e. families Methanobacteriaceae, Haloferacaceae and Methanomethylophilaceae, are the discriminant factors determining the distance between samples (Fig. 3a).

The gut bacterial communities in the human gastrointestinal tract have been partitioned into several clusters. Each cluster (enterotype) is overrepresented by a distinct set of bacterial genera [27]. As shown in the taxonomic and clustering analyses above, the community composition and structure of the Korean gut archaeome highlighted the importance of the several robust clusters that were prevalent across samples with different abundances. Next, we assessed the presence of enterotypes by applying partitioning around medoids (PAM) clustering analysis to the Bray-Curtis dissimilarity matrix generated from the family-level relative abundance profiles (Fig. 3b). The results showed four distinct clusters: Methanobacteriaceae as archaeal enterotype (MBA enterotype), Methanomethylophilaceae as archaeal enterotype (MMA enterotype), Haloferacaceae as archaeal enterotype (HFA enterotype) and the unclassified Euryarchaeota related to uncultured phylotypes in genus Methanosphaera or Haloplanus as archaeal enterotype (UEA enterotype; Fig.
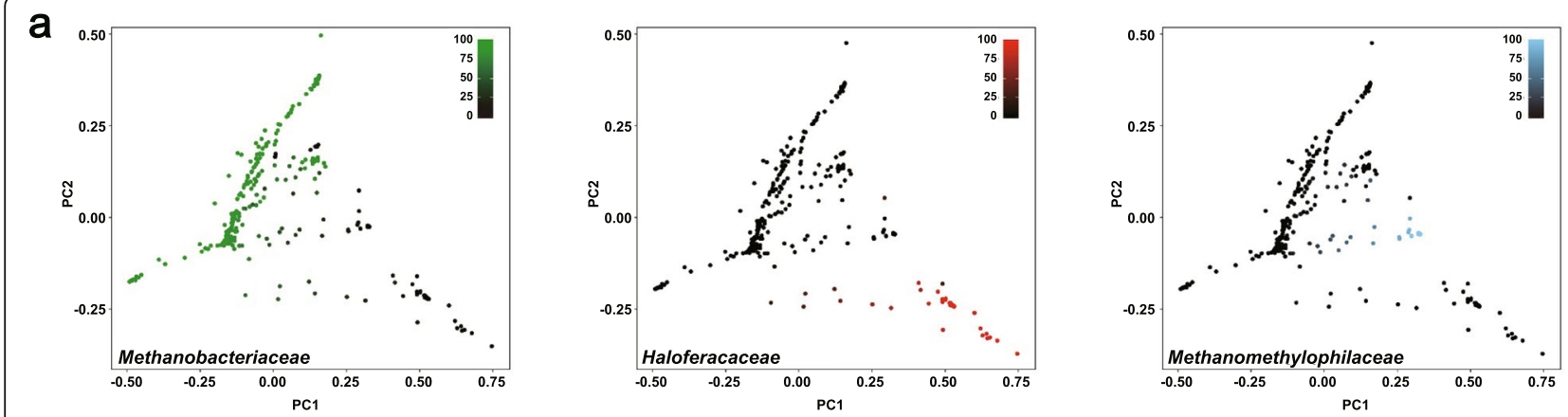

b

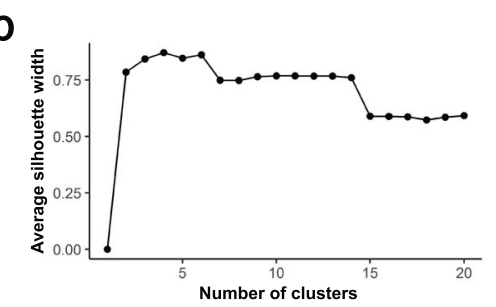

d

Cluster
1
2
3
4
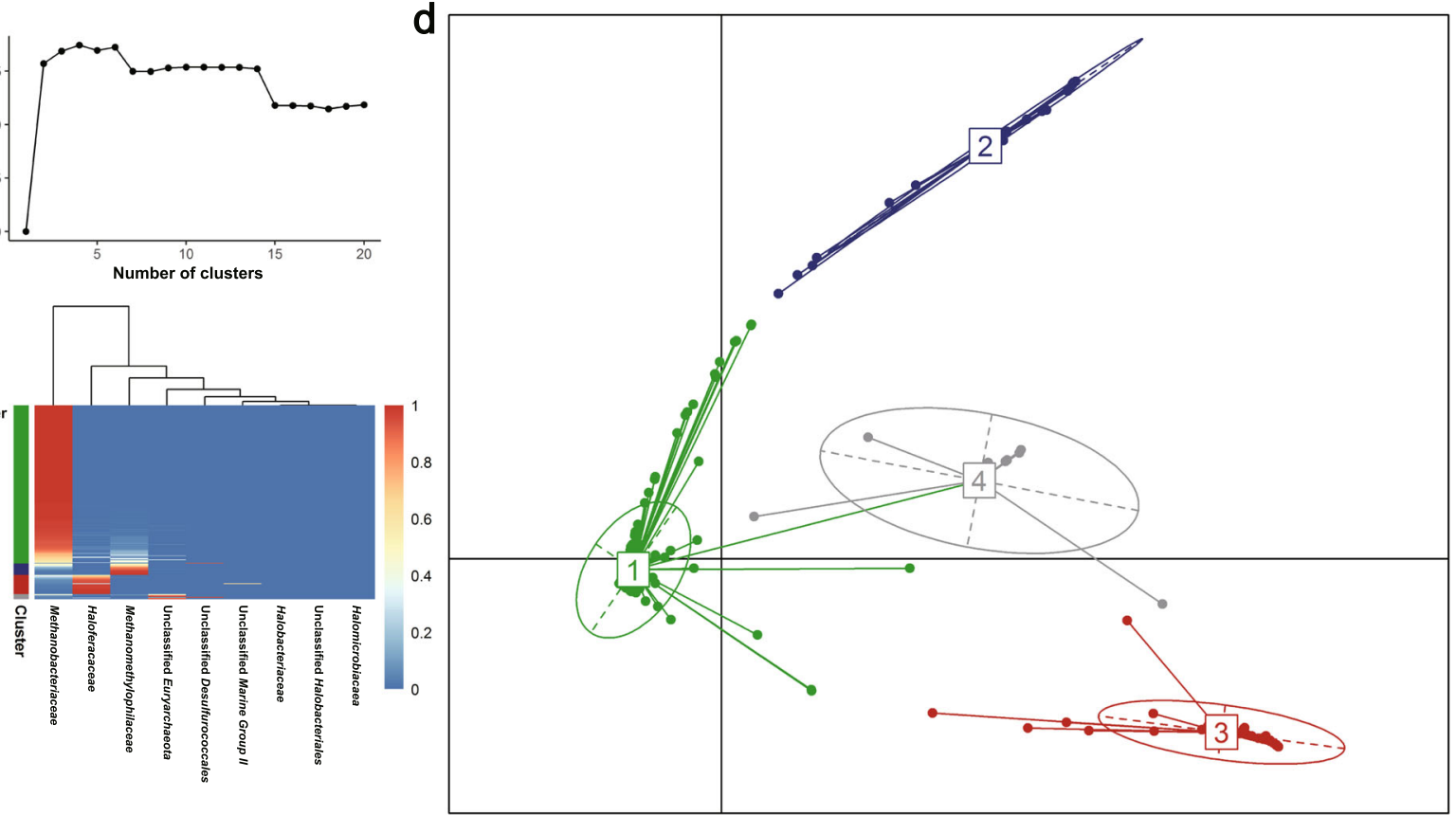

Fig. 3 Identification of enterotypes in the Korean gut archaeome. The faecal archaeal 16S rRNA gene sequences were prepared from Korean individuals. We subsampled 342 from the 381 archaeal-sequence-positive samples at a sampling depth of 10,000. a PCoA was generated based on the weighted UniFrac distance matrix of the archaeal $16 \mathrm{~S}$ rRNA gene sequence data. Colour gradations show the relative abundances of the families Methanobacteriaceae (green), Haloferacaceae (red) and Methanomethylophilaceae (blue). b-d. Assortment of gut archaeal communities into enterotypes. b The optimal number of clusters was estimated at the family level by partitioning around medoids (PAM) clustering based on the Bray-Curtis dissimilarity matrix. c The relative abundance of the archaeal communities in all samples are presented according to the clusters and shown as a heat map. $\mathbf{d}$ Visualisation of enterotypes. The dots and numbers represent the abundance distributions of the archaeal taxa from individual samples and the centre of each enterotype, respectively 
$3 c, d)$. These suggested that the distinct set of overrepresented taxa (enterotypes) might be the consequence of a well-balanced symbiotic relationship between the host and archaea in the gut environment.

\section{Diversity of haloarchaeal phylotypes in the human gut} Phylogenetic analysis of the methanogen-assigned sequences revealed three clusters of the methanogen phylotypes with the genera Methanobrevibacter (318 ASVs) and Methanosphaera groups (178 ASVs) and the family Methanomethylophilaceae group (44 ASVs; see Additional file 1: Supplementary Fig. S3). Studies on human gut archaeomes dominated by methanogens have been already described elsewhere [12, 17, 22, 28]. Therefore, we analysed the haloarchaea-assigned sequences, which have not yet been reported in the metataxonomic studies of the human gut archaeome. To evaluate the diversity of the haloarchaeal phylotypes in the human gut, we conducted a phylogenetic analysis based on the 139 haloarchaea-assigned ASVs with trimmed 16S rRNA gene sequences of the validated haloarchaeal species and public clonal sequences. We observed that the majority of haloarchaeal phylotypes are closely related to the genera Haloplanus, Halolamina and Halorubrum (Fig. 4a). In particular, we observed three branched clusters of the haloarchaeal phylotypes, which were relatively distantly located from the validated haloarchaeal species. The Haloplanus subgroup consisted of 68 ASVs (Fig. 4b); Halolamina, 32 ASVs (Fig. 4c); and Halorubrum, 13 ASVs (Fig. 4d). We next assessed whether sequences assigned to haloarchaea occur in other sample cohorts by trawling the publicly available human metagenomic and metataxonomic datasets bases using the EBI MGnify. As shown in Table 1, we found several studies possessing the metagenomic and metataxonomic sequences assigned to haloarchaea, implying that the human gut is capable of harbouring diverse and metabolically unknown haloarchaeal strains.

A positive correlation between the richness of the halophilic bacteria and faecal salinity has been recently reported [19]. To verify this association, we randomly selected 20 faecal samples with different percent relative abundances of haloarchaea, i.e. 5.38-99.33\%, and measured faecal salinity using a salinity refractometer, yielding a mean salinity of $0.68 \%$, ranging from 0.30 to $1.05 \%$ (see Additional file 1: Supplementary Fig. S4a). Correlation coefficient and linear regression analyses revealed no association between the relative abundance of haloarchaea and faecal salinity. Further, we analysed the inorganic elements mainly consisting of the salt (e.g. sodium, potassium, magnesium and calcium) of 20 selected samples using an inductively coupled plasmamass spectrometer (ICP-MS). On average, each inorganic element possessed less than $1 \%$ of total faecal weight: $0.04 \%, 0.40 \%, 0.24 \%$ and $0.50 \%$ for sodium, potassium, magnesium and calcium, respectively (see Additional file 1: Supplementary Fig. S4b). Similar to the salinity data, no positive or negative relationship was found between the relative abundance of haloarchaea and the relative amount of faecal inorganic elements.

\section{Detection of haloarchaea in the human gut by fluorescence in situ hybridisation}

We attempted to verify the presence of haloarchaea in the human gut by a non-sequencing-based approach using the FISH analysis. We designed a haloarchaeaspecific probe (HALO775) and tested the specificity of the oligonucleotide probe. Neither the undesired match (i.e. in silico binding of the HALO775 with nonhaloarchaeal taxa, such as methanogen, bacteria and eukaryotes; see Additional file 2: Supplementary Tables S1 and S2) nor the unspecific binding of the HALO775 cy3 with the cultured bacterium (Escherichia coli K12) and other archaeon (Methanobrevibacter smithii JCM $30028^{\mathrm{T}}$ ) was found (Fig. 5a). A positive signal of the HALO775 $5_{\text {cy3 }}$ was only observed with the cultured haloarchaeon (Haloplanus salinus JCM $18368^{\mathrm{T}}$ ). Based on both the total archaeal abundance and the metataxonomic data, we selected three faecal samples that possess a different proportion of the haloarchaea-assigned sequences (sample J0885, J0111 and J0054; Fig. 2). The FISH analysis successfully detected a positive signal for haloarchaea from the selected samples (Fig. 5b). These results collectively suggest the presence of haloarchaea in the human gut, which was detected by sequencingbased (i.e. metataxonomics) and non-sequencing-based (i.e. FISH) methods.

\section{Correlation analysis of the gut archaeal profiles with host factors}

We next evaluated the effect of host factors on the community structure of the Korean gut archaeome by conducting correlation coefficient analysis using two variables: relative abundance of the proportionally abundant archaeal taxa at the genus level and host factors, including dietary nutrients, clinical phenotypes and food categories. We observed strong negative/positive correlations (Spearman's rank correlation analysis, adjusted $P<$ 0.05 ) between several archaeal taxa and dietary nutrients: the genus Halorubrum was negatively correlated with calcium, potassium, vitamin $\mathrm{A}$, vitamin $\mathrm{B}_{6}$, vitamin $\mathrm{C}$, folate, carotene and fibre levels; the genus Methanosphaera was positively correlated with energy, protein, fat, phosphorus, iron, potassium, vitamin $B_{1}$, vitamin $B_{2}$, zinc, ash, vitamin $\mathrm{E}$ and cholesterol levels (Fig. 6 left). In host clinical phenotypes, the genera Halorubrum and Halobacterium showed significantly negative correlation (adjusted $P<0.05$ ) with several lipids, i.e. total 


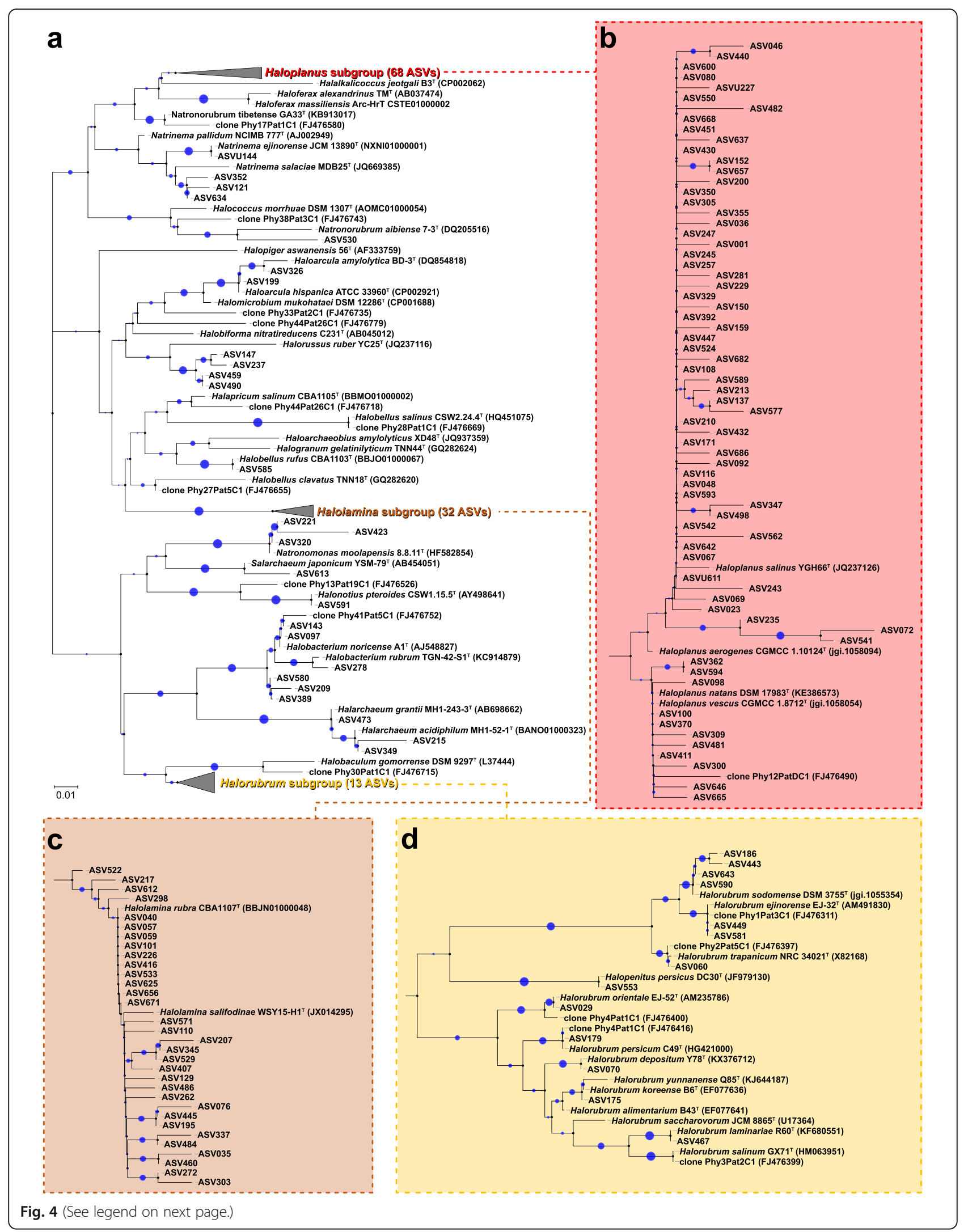


(See figure on previous page.)

Fig. 4 Phylogenetic analysis of the amplicon sequence variants (ASVs) of haloarchaea. The ASVs assigned to the haloarchaea were identified in the faecal archaeal 16S rRNA gene sequence data of the 381 Koreans. The 16S rRNA gene sequences of the validated haloarchaeal species and public clonal sequences were included. a A phylogenetic consensus tree based on the $16 \mathrm{~S}$ rRNA gene sequences was reconstructed using the neighbour joining algorithm, indicating the taxonomic positions of the ASVs. b-d Phylogenetic trees of the three subgroups are shown:

Haloplanus subgroup (b), Halolamina subgroup (c) and Halorubrum subgroup (d). The size of blue circles represents the bootstrap value based on 1000 replications. Bar, 0.01 accumulated changes per nucleotide

cholesterol (TotalC) and low-density lipoprotein cholesterol (LDLC). In addition, we observed a positive correlation (adjusted $P<0.05$ ) between the genus Halolamina and renal functions (estimated glomerular filtration rate using the MDRD formula, eGFR_MDRD and estimated glomerular filtration rate using the CKD-EPI formula, eGFR_CKDEPI; Fig. 6, right). We also found several positive/negative correlations of the haloarchaeal taxa with food categories (see Additional file 1: Supplementary Fig. S5). However, these results implied no common (dietary) factor influencing the abundance of haloarchaea in the human gut.

\section{Comparison of the human gut archaeome with the gut archaeomes of the great apes}

We observed the assortative characteristics of the four distinct enterotypes (Fig. 3d). As the host diet might affect the formation of enterotypes [29], the archaeal enterotypes may have arisen over relatively recent timescales in the human lineage. However, Fig. 6 and Additional file 1: Supplementary Fig. S5 show weak (or no) relationship between archaeal abundances and host dietary factors. Next, we assessed whether these enterotypes are the results of more ancient features (such as host immune system and gut physiology) that might be derived before or during the diversification of the great ape species by comparing the Korean gut archaeome with other human gut archaeomes $[11,28]$ and those of the great apes, including orangutan, gorilla, chimpanzee and bonobo [30]. PCoA of the Bray-Curtis dissimilarity matrix showed the typical horseshoe shape of all merged samples (see Additional file 1: Supplementary Fig. S6a), indicating high dissimilarity among the gut archaeomes of the great apes. Comparison of the human and nonhuman samples revealed that weighted PCoA (see Additional file 1: Supplementary Fig. S6a) and unweighted PCoA (based on the Jaccard dissimilarity matrix; see Additional file 1: Supplementary Fig. S6b) showed significantly separated community structure and composition of the gut archaeome, respectively (permutational multivariate analysis of variance [PERM ANOVA], $P=0.001$, comparison between the human and non-human samples). UPGMA clustering analysis showed that the community structure of the gut archaeome of the great apes (except those of humans) appeared to mimic the host phylogeny, as shown by the absence of any significant difference in the distance between chimpanzee and bonobo gut archaeome (see Additional file 1: Supplementary Fig. S6c). Neither the community structure nor the composition of the human gut archaeome was closely related to those of chimpanzees or bonobos (see Additional file 1: Supplementary Fig. S6c and d), suggesting that the human gut archaeome is distinct and not related to host phylogeny.

\section{Discussion}

Here, we reported the human gut archaeome of 897 Korean subjects. Using archaeal metataxonomic analysis with nested PCR amplicons and an archaea-specific primer set, we were able to detect archaeal sequences from 381 samples (42.47\%, Fig. 1). This distribution ratio of the archaeal 16S rRNA gene sequence-positive samples is in accordance with the results of a previous study showing archaeal colonisation in the gastrointestinal tract of approximately half of the human population [31]. Furthermore, we observed the abundance of the human gut archaea $(10.24 \pm 4.58 \%$ of the total bacterial and archaeal abundance; Fig. 2). Archaeal abundance in the human gut has been estimated previously, ranging from 0.1 to $21.3 \%$ [11, 32]. Our abundance estimation did not vary substantially from those of previous studies, although the values were based on a relatively larger sample size $(n=150)$, thereby enhancing the reliability of the estimation. Both the prevalence and the quantitative data obtained collectively supported the robustness of our methodological approach, which minimised the experimental pitfalls in human archaeome analysis. Given that the adjusted value of the gut archaeal abundance based on the archaeal/bacterial $16 \mathrm{~S}$ rRNA gene copy number per genome showed over a twofold increase in estimated abundance of the human gut archaea (Fig. 2), the quantitative characteristics of the gut archaeome and their net effect(s) on host physiology remain to be elucidated.

PCR amplicon sequencing analysis revealed that sequences assigned to the genus Methanobrevibacter and Methanosphaera are most proportionally abundant in all faecal samples where archaea are detected. These two genera contain strictly anaerobic methanogens utilising hydrogen/carbon dioxide and hydrogen/methanol, respectively [17], and are capable of entering into syntrophic relationships with gut bacterial microbiota by 
Table 1 Haloarchaea-assigned sequences in other publicly available metagenomic and metataxonomic studies

\begin{tabular}{llll}
\hline $\begin{array}{l}\text { Accession } \\
\text { number }\end{array}$ & Study type & $\begin{array}{l}\text { Amplified } \\
\text { region }\end{array}$ & Study name \\
\hline ERP001956 Metagenomic & N/A & $\begin{array}{l}\text { Diagnostic Metagenomics: A Culture-Independent } \\
\text { Approach to the Investigation of Bacterial Infections }\end{array}$ \\
SRP001634 Metagenomic & N/A & Microbial composition of samples from infant gut
\end{tabular}

SRP001634 Metagenomic N/A Microbial composition of samples from infant gut

Haloarchaea-assigned taxon

c__Halobacteria; O__Halobacteriales; f__ Halobacteriaceae

\begin{abstract}
SRP073172 Metagenomic N/A
SRP096283 Metagenomic N/A
\end{abstract}

SRP118697 Metagenomic N/A

SRP128128 Metagenomic N/A

ERP015450 Metagenomic N/A

ERP005883 Metataxonomic V4

ERP010229 Metataxonomic V4

ERP021093 Metataxonomic V4

$$
\text { Microbial composition of samples from infant gut }
$$

Dysbiosis of gut microbiota contributes to the pathogenesis of hypertension

Effects of cholera on the human gut microbiota, and interactions between human gut microbes and Vibrio cholera

Gut microbial succession follows acute secretory diarrhea in humans

Gut microbiome from patients obtained by 16s rRNA sequencing
c_Halobacteria; O__Halobacteriales; f__ Halobacteriaceae; g_; s_ c_Halobacteria; O__Halobacteriales; f__ Halobacteriaceae; g__Halococcus; s c_Halobacteria; O__Halobacteriales; $f$ Halobacteriaceae; g__Halorhabdus; s_

DNA from FIT can replace stool for microbiota-based colorectal

c__Halobacteria; O__Halobacteriales; f__ Halobacteriaceae; g_; s_

Human gut metagenome and metatranscriptome raw c__Halobacteria

Dynamics of human gut microbiota and metabolites in response to prebiotic interventions

c_Halobacteria; O__Halobacteriales

c__Halobacteria

C_Halobacteria; O__Halobacteriales c_Halobacteria; O__Halobacteriales; f__ Halobacteriaceae; g_Halobacterium c_Halobacteria; O__Halobacteriales; f__ Halococcaceae; g__Halococcus c__Halobacteria; O__Natrialbales; f__Natrialbaceae; g_Natronococcus

c_Halobacteria; O__Halobacteriales; f__ Halobacteriaceae; g__Haloarcula; s_

c_Halobacteria; O__Halobacteriales; f__ Halobacteriaceae; $g_{\text {_. }}$ s_

c_Halobacteria; O__Halobacteriales; f__ Halobacteriaceae; g_Halorubrum; s_-

c_Halobacteria; O__Halobacteriales; f__ Halobacteriaceae; g__Haloterrigena; s_

c_Halobacteria; O__Halobacteriales; f__ Halobacteriaceae; $g_{\text {_. }}$ s_

c__Halobacteria; O__Halobacteriales; f__ Halobacteriaceae; g__Halalkalicoccus; s_jeotgali

c_Halobacteria; O__Halobacteriales; f__ Halobacteriaceae; g__Halococcus; s_

c_Halobacteria; O__Halobacteriales; f__ Halobacteriaceae; g__Halococcus; s_ hamelinensis

c__Halobacteria; O__Halobacteriales; f__ Halobacteriaceae; g_Halorhabdus; s_

c_Halobacteria; O__Halobacteriales; f__ Halobacteriaceae; g__Haloterrigena; s_

C__Halobacteria; O__Halobacteriales; f__ Halobacteriaceae; g__Natronococcus; s_

c_Halobacteria; O__Halobacteriales; f__ Halobacteriaceae; $g_{\text {_. }}$ s_

c_Halobacteria; O__Halobacteriales; f__ Halobacteriaceae; g__GA41; s_

c_Halobacteria; O__Halobacteriales; f__ Halobacteriaceae; g__Haloarcula; s_

c_Halobacteria; O__Halobacteriales; f__ Halobacteriaceae; 9__Halobacteriaceae; s_GX3

c__Halobacteria; O__Halobacteriales; f__ Halobacteriaceae; g__Halobacterium; s_ c_Halobacteria; O__Halobacteriales; f__ Halobacteriaceae; g__Halococcus; s_ 
Table 1 Haloarchaea-assigned sequences in other publicly available metagenomic and metataxonomic studies (Continued)

\begin{tabular}{|c|c|c|c|c|}
\hline $\begin{array}{l}\text { Accession } \\
\text { number }\end{array}$ & Study type & $\begin{array}{l}\text { Amplified } \\
\text { region }\end{array}$ & Study name & Haloarchaea-assigned taxon \\
\hline & & & & $\begin{array}{l}\text { c_Halobacteria; o__Halobacteriales; f__ } \\
\text { Halobacteriaceae; g_Halomicrobium; s__ }\end{array}$ \\
\hline & & & & $\begin{array}{l}\text { C_Halobacteria; o__Halobacteriales; } f_{-} \\
\text {Halobacteriaceae; g_Halomicrobium; }{ }_{\text {__ }} \\
\text { mukohataei }\end{array}$ \\
\hline & & & & $\begin{array}{l}\text { C_Halobacteria; O_Halobacteriales; f__ } \\
\text { Halobacteriaceae; g__Halonotius; s__ }\end{array}$ \\
\hline & & & & $\begin{array}{l}\text { c_Halobacteria; o__Halobacteriales; } f \text { __ } \\
\text { Halobacteriaceae; g__Haloplanus; s__ }\end{array}$ \\
\hline & & & & $\begin{array}{l}\text { c_Halobacteria; o__Halobacteriales; } f_{-} \text {__Halobacteriaceae; } g_{\text {___Haloquadratum; }} \text { __ }\end{array}$ \\
\hline & & & & $\begin{array}{l}\text { C_Halobacteria; O__Halobacteriales; f__ } \\
\text { Halobacteriaceae; g__Halorhabdus; s__ }\end{array}$ \\
\hline & & & & $\begin{array}{l}\text { c_Halobacteria; o_Halobacteriales; } f \text { _- } \\
\text { Halobacteriaceae; g__Halorubrum; s_- }\end{array}$ \\
\hline & & & & $\begin{array}{l}\text { c_Halobacteria; O__Halobacteriales; } f \text { ___ } \\
\text { Halobacteriaceae; g__Natronomonas; } \text { s_ }_{\text {_. }}\end{array}$ \\
\hline & & & & $\begin{array}{l}\text { C_Halobacteria; O_Halobacteriales; } f \text { _- } \\
\text { Halobacteriaceae; g__XKL75; s__ }\end{array}$ \\
\hline & & & & C__Halobacteria; O__Halobacteriales; f_MSP41; g__ \\
\hline \multirow[t]{4}{*}{ ERP021896 } & Metataxonomic & V4 & Moving pictures of the human microbiome & C__Halobacteria; O__Halobacteriales \\
\hline & & & & $\begin{array}{l}\text { C_Halobacteria; o__Halobacteriales; } f \text { _ } \\
\text { Halobacteriaceae }\end{array}$ \\
\hline & & & & $\begin{array}{l}\text { c_Halobacteria; o_Halobacteriales; } f \text { _- } \\
\text { Halobacteriaceae; g__Halarchaeum }\end{array}$ \\
\hline & & & & $\begin{array}{l}\text { c_Halobacteria; o_Halobacteriales; } f \text { _- } \\
\text { Halobacteriaceae; g__Halobacterium }\end{array}$ \\
\hline \multirow[t]{5}{*}{ ERP107577 } & Metataxonomic & V3orV4 & LogMPIE: Landscape Of Gut Microbiome - Pan & c__Halobacteria \\
\hline & & & India Explo & c__Halobacteria; O__Haloferacales \\
\hline & & & & $\begin{array}{l}\text { C_Halobacteria; O__Haloferacales; f__ } \\
\text { Haloferacaceae }\end{array}$ \\
\hline & & & & $\begin{array}{l}\text { C_Halobacteria; O_Haloferacales; } f \text { __ } \\
\text { Halorubraceae; g__Haloparvum }\end{array}$ \\
\hline & & & & c_Halobacteria; O__Natrialbales; f__Natrialbaceae \\
\hline ERP109659 & Metataxonomic & V3-V4 & $\begin{array}{l}\text { Gut microbiota in Parkinson's disease: temporal } \\
\text { stability and disease progression }\end{array}$ & c_Halobacteria; O__Halobacteriales \\
\hline
\end{tabular}

N/A not applicable

consuming the excess hydrogen produced during anaerobic fermentation of carbohydrates, thereby increasing the ATP synthesis of anaerobic bacteria and promoting the growth of gut bacteria [12]. In contrast, gut methanogens have been correlated with various diseases (i.e. colorectal cancer, obesity, anorexia, inflammatory bowel disease, irritable bowel syndrome, diverticulosis, constipation and periodontitis) in humans [33-35]; but the results are inconsistent, and are dependent on the methodology used. Correlation coefficient analysis on the abundance of methanogens and host clinical phenotypes did not show any relationship between the two variables. We instead observed that the genus
Methanosphaera was positively related to host dietary nutrients, suggesting that the abundance of some specific members of the human gut archaeome might be orthogonally manipulated by host dietary nutrients.

In particular, this study demonstrated the extensive colonisation of the Korean gut by haloarchaea. We detected haloarchaea-assigned phylotypes in 364 out of 381 archaea-positive faecal samples (95.54\%). Haloarchaea were more prevalent than methanogens in some samples. To our knowledge, no metataxonomic study has reported haloarchaea-assigned sequences in the human gut. A recent study by Oxley et al. reported the possible presence of haloarchaea in the human intestinal 


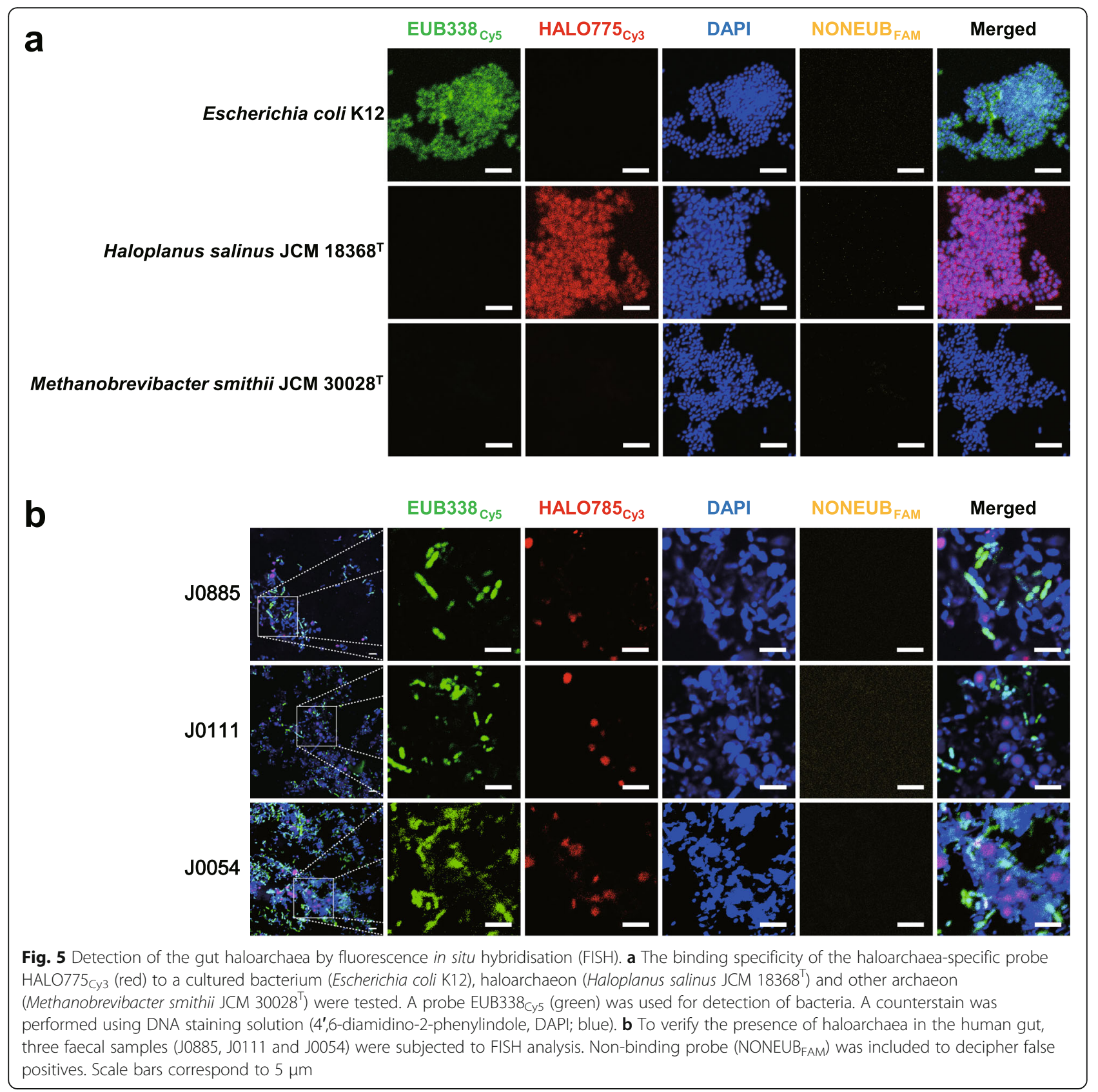

mucosa, although haloarchaea has not yet been confirmed as indigenous gut microbiota because (i) the results were based on an insufficient number of sequences obtained from the clone libraries, and (ii) the study was biased toward the diseased samples (i.e. they obtained mucosal biopsies from the colons of patients with inflammatory bowel disease) [36]. Using a culturedependent approach, Seck et al. recently isolated two haloarchaeal strains from 572 human faecal samples. They subsequently conducted metataxonomic analysis using the archaeal 16S rRNA gene amplicon sequencing of 164 human faecal samples but did not detect haloarchaea-assigned sequences [19].

Haloarchaea includes salt-loving microorganisms, which have been considered extremophiles as they were frequently detected in hypersaline environments. The genus Haloplanus consists of eight species isolated from solar salterns, aquaculture farms, crude solar salts, and Dead Sea-Red Sea water mixtures [37]. They can grow in medium containing $0.9-5.1 \mathrm{M} \mathrm{NaCl}$, and some species grow anaerobically in the presence of nitrate, dimethylsulphoxide (DMSO) or L-arginine. The genus Halorubrum consists of 37 species isolated from 


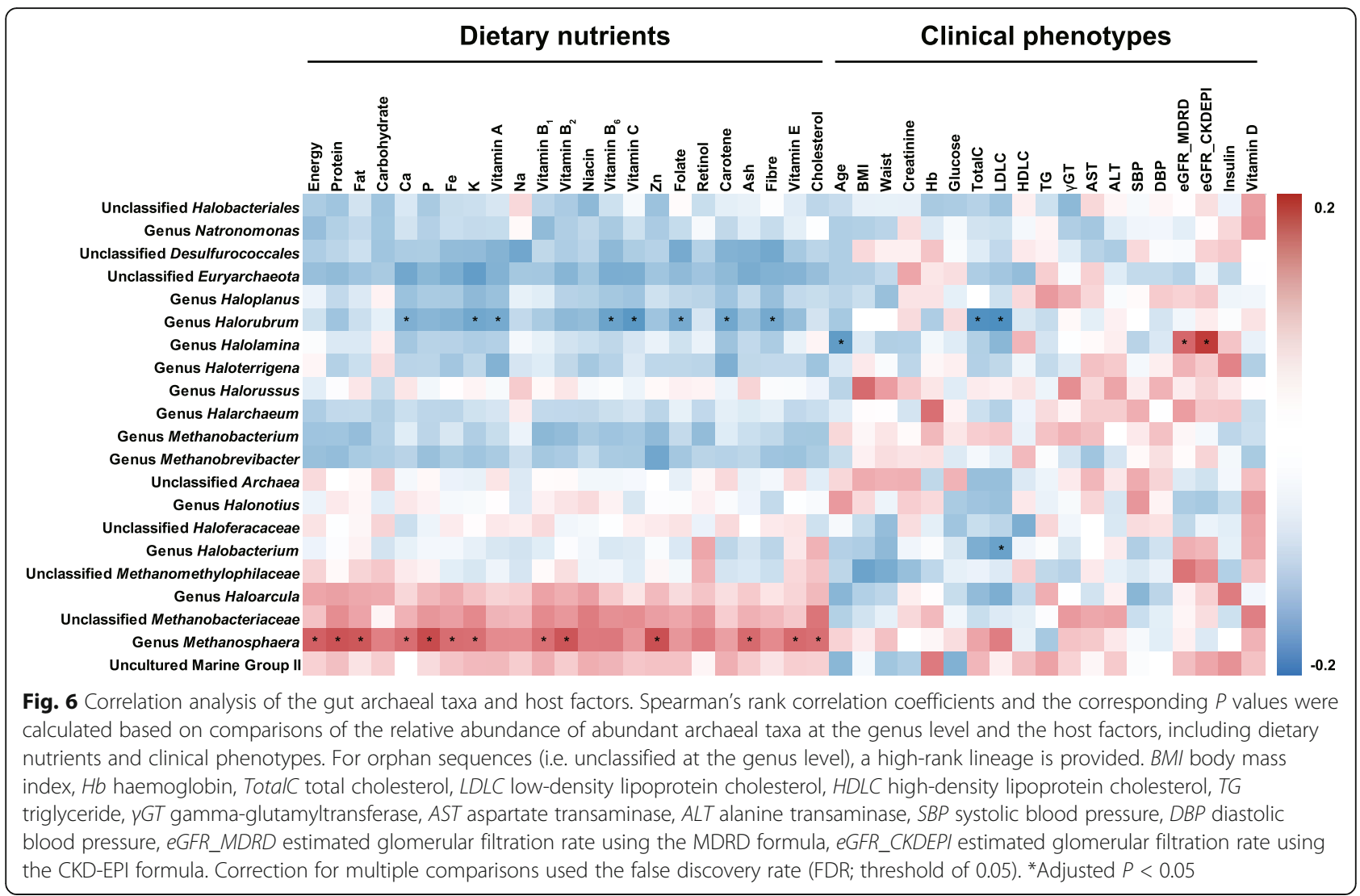

hypersaline environments, such as rock salt and solar saltern [38]. They grow in medium with $1.0-5.2 \mathrm{M} \mathrm{NaCl}$ and some species grow under anaerobic conditions in the presence of nitrate, DMSO or L-arginine. Various haloarchaeal strains were also isolated from food products and ingredients of salt-fermented food products [39]. In Korea, salt is widely used in fermented foods as the oldest food preservation technique for inhibiting the growth of unfavourable bacteria. Hence, we attempted to identify the factor(s) affecting the high prevalence/ abundance of gut haloarchaea from dietary habits; however, no positive relationship was observed between the relative abundance of haloarchaea and host dietary nutrients/salt-containing food items (Fig. 6 and Supplementary Fig. S5). This was not surprising as fermented food products are daily consumed by billions of people worldwide [40]. Therefore, investigations regarding the mechanisms underlying haloarchaeal dissemination in the human gut are essential.

We next investigated if the gut haloarchaea are members of the human natural gut microbiota or if they reflect foodborne microbiota that transiently passes through the gut. From the metataxonomic and FISH analyses, we could not confirm whether the observed haloarchaea are colonisers of the gut, merely passing through after environmental exposure, or were consumed in the diet. Given that Korean guts possessed a structured community of haloarchaea with a complex composition of haloarchaeal phylotypes (Figs. 3 and 4), the possible growth and development of haloarchaea in the human gut should be investigated further. As shown in Additional file 1: Supplementary Fig. S4, the mean faecal salinity in Korean subjects was $0.68 \%$, ranging from 0.30 to $1.05 \%$. Haloarchaea is increasingly detected in habitats of relatively low salinities [41, 42], supporting the possible growth of haloarchaea in the human gut. Higher faecal salinity might not necessarily promote haloarchaeal growth in the human gut, as evidenced by the absence of haloarchaea-assigned sequence, in which the mean salinity is $0.7 \%$ and ranging from 0 to $6 \%$ [19]. Members of haloarchaea can grow either aerobically or anaerobically. The nutritional demands and metabolic pathways for aerobic heterotrophic metabolism of haloarchaea are diverse [43]. However, the normal human gut is characterised by anaerobic conditions with extremely low quantity of oxygen in the luminal space maintained by the oxygen consumption of the host cells $[44,45]$. Oxygen has low solubility in salt-saturated natural environments and is possibly a limiting resource for haloarchaea growth. Under these conditions, many haloarchaeal members grow as facultative anaerobic heterotrophs using alternative electron acceptors (such 
as nitrate, trimethylamine $\mathrm{N}$-oxide, fumarate, thiosulphate or elemental sulphur) [43] for metabolism and fermentation of L-arginine, as well as using strictly anaerobic acetotrophic metabolism with acetate as the electron donor and elemental sulphur as the electron acceptor [46], and strictly anaerobic lithoheterotrophic metabolism with hydrogen as the electron donor and elemental sulphur or thiosulphate as electron acceptor [47]. A culture-dependent approach, along with confirmation of the existence of the haloarchaeal phylotypes based on culture-independent methods, combined with the use of a gnotobiotic animal model (i.e. animals harbouring a defined haloarchaeal communities in their gut) will further elucidate the growth and development of anaerobic heterotrophic or undiscovered ecotypic haloarchaea in the gut environment.

We also categorised the Korean gut archaeome into four archaeal enterotypes: the Methanobacteriaceae-, Methanomethylophilaceae-, Haloferacaceae- and the unclassified Euryarchaeota-dominant archaeome (Fig. 3). These enterotypes reflect the proportional categories (or patterns) within the gut microbiota in different individuals, thereby being the well-balanced and quite stable microbial members among all individuals [27]. However, a careful interpretation is needed as the categorisation of the gut microbiota based on the dominance of certain genera may result in oversimplification [48]. Further studies to clarify the archaeal complexity within the gut and to link it to clinical traits will be of interest.

\section{Conclusions}

We conducted a population-level metataxonomic analysis on the gut archaeome of Koreans. Our deep sequencing analysis revealed unexpectedly diverse archaeal communities in the human gut. Interestingly, we observed extensive colonisation of haloarchaea in the Korean gut. However, our study has some limitations; we could not show that the abundant archaeal species belonging to both the methanogens and haloarchaea (identified from metataxonomic analysis) consisted of members of the viable natural gut microbiota. Further identification of the spatiotemporal dynamics of the human gut archaeome using longitudinal samples from different compartments of the intestinal tract should be performed. In addition, the clustering results of the archaeal communities in human and non-human samples (shown in Additional file 1: Supplementary Fig. S6) might be biased due to methodological differences used in each study. Nonetheless, we observed (i) an unexpectedly diverse archaeal community in the human gut and (ii) inter-individual variation in the human gut archaeome characterised by the presence of several clusters of gut archaeal enterotypes. Collectively, our results have expanded our understanding of the human gut archaeome, suggesting that the human gut archaeome is indigenous, responsive, and functional.

\section{Methods \\ Sample preparation and DNA extraction}

A total of 897 subjects were enrolled in the Korean gut microbiome project. Subjects who had consumed antibiotics in the 3 months before the study or those with a history of major gastrointestinal diseases were excluded. Subjects consisted of 203 males and 694 females, and their age ranged from 20 to 90 . All subjects completed a questionnaire that covers comprehensive demographic and lifestyle information and a food frequency questionnaire (FFQ) [49]. Subjects underwent blood biochemical tests and anthropometrical measurements. Metadata, including clinical measurements and food frequency details, are available in the independent study by Lim et al. [50]. Participants' faecal samples were collected in both an OMNIgene-GUT tube (DNA Genotek, Ontario, Canada) and a sterile stool collection container. Microbial DNA was extracted using the QIAamp DNA stool mini kit (QIAGEN, Hilden, Germany) with additional bead-beating and heating steps [51]. The elution buffer volumes were $200 \mu \mathrm{L}$, and the extracted DNA samples were stored at $-20{ }^{\circ} \mathrm{C}$ until further use.

\section{Library preparation and sequencing of archaeal 16S rRNA gene}

To amplify the archaeal $16 \mathrm{~S}$ rRNA genes, a nested PCR was performed as described previously [28] using MG 2X PCR mastermix (MGmed, Seoul, South Korea) and Accupower $^{\text {Ts }}$ Hotstart PCR premix (Bioneer, Daejeon, South Korea) in the first ( 25 cycles) and second PCRs (25 cycles), respectively. Briefly, we used the primer pairs S-D-Arch-0344-a-S-20 and S-D-Arch-0911-a-A-20 in the first PCR, and S-D-Arch-0349-a-S-17 and S-D-Arch0519-a-A-16, which included the Illumina adapter sequence, in the second PCR. The primer sequences are listed in Additional file 2: Supplementary Table S3. Three PCR products with the same template were pooled. To purify the products obtained in the first and second rounds, $x$-tracta ${ }^{\text {Tu }}$ Gel Extractor (Promega, Madison, WI, USA) and Qiaquick PCR \& gel cleanup kit (QIAGEN) were used. Among the 897 samples, 381 were confirmed using electrophoresis on $1 \%$ agarose gel. To verify the size of the PCR-enriched fragments, template size distribution was checked using the Agilent Technologies 2100 bioanalyzer and a DNA 1000 chip. Libraries for archaeal 16S rRNA gene sequences were constructed using the Nextera DNA Flex library preparation kit (Illumina, San Diego, CA, USA), following the manufacturer's instruction. A subsequent limited-cycle amplification step was performed to add multiplexing indices and Illumina sequencing adapters. The final 
products were normalised and pooled using PicoGreen, and the size of the libraries was verified using the TapeStation DNA screentape D1000 (Agilent, Santa Clara, CA, USA). Sequencing was performed using the HiSeq $^{\text {Ti }} \mathrm{X}$ platform (Illumina). We also investigated possible DNA contamination in all reagents used for DNA extraction. PCR analysis targeting the archaeal $16 \mathrm{~S}$ rRNA gene $(25+25$ cycle reactions) revealed no apparent contamination (Additional file 1: Supplementary Fig. S7a). PCR amplicons from 'blank' negative DNA extraction/PCR controls (i.e. PCR products of template acquired from a sham extraction to which no faecal sample is added; $n=3$ ) were used as the negative controls (Additional file 1: Supplementary Fig. S7b).

\section{Analysis of 165 rRNA gene sequence data}

By using the bcl2fastq2 conversion software v. 2.20.0. (Illumina), the adapter sequences were trimmed from the raw FASTQ reads, and the trimmed reads were demultiplexed according to the samples. To generate the ASVs feature table, the sorted reads were imported and processed using QIIME2 v. 2018.11 [52]. In total, 275, 909,328 imported paired reads were quality filtered, denoised and merged using the DADA2 plugin [53]. Chimeric sequences and singleton ASVs were excluded in further analyses. Rarefaction curves were constructed using plugin diversity alpha-rarefaction in QIIME2. For taxonomic classification, we used plugin q2-featureclassifier, the classify-sklearn method [54] and the pretrained SILVA v. 132 database [55] with 99\% identity. An overview of the human gut archaeal 16S rRNA gene sequence dataset is provided in Additional file 2: Supplementary Table S4.

To calculate the 16S rRNA gene similarities with the secondary structure, the ASVs were aligned using the RDP aligner (https://pyro.cme.msu.edu/aligner/form. spr). Afterwards, the aligned ASVs were used to construct a phylogenetic tree using the neighbour joining algorithm based on the Kimura 2-parameter model with 1000 bootstraps in MEGA X [56]. Phylogenetic tree visualisation was performed using iTOL v. 5 [57]. Phylogenetic analysis of the haloarchaea-assigned sequences included 16S rRNA gene sequences of the type strains belonging to the class Halobacteria and clone sequences from Oxley et al. [36], whereas that of the methanogen-assigned sequences included $16 \mathrm{~S}$ rRNA gene sequences of the type strains belonging to the genera Methanobrevibacter and Methanosphaera and the family Methanomethylophilaceae. To determine the species diversity in each human faecal sample, alpha and beta diversity analyses were performed using the plugin q2-diversity in QIIME2 v. 2018.11. Based on rarefaction results, we subsampled the sequences at a sampling depth of 10,000 (Additional file 1: Supplementary Fig.
S1) and included 342 from the 381 archaeal sequencepositive samples. Subsequently, group difference was determined based on metadata.

Archaeal enterotypes were identified as previously described [58]. Briefly, the samples were clustered using PAM clustering [59] with the Bray-Curtis dissimilarity matrix [60] at the family level. The optimal number of clusters was determined using the silhouette index [61]. Enterotypes were visualised by the PCoA plot. To find the sequences assigned to haloarchaea in other sample cohorts, we trawled both the publicly available human metagenomic and metataxonomic datasets using the EBI MGnify [62]. All the data were obtained from the EBI MGnify database.

The archaeal 16S rRNA dataset generated using HiSeq for the negative controls is summarised in Additional file 2: Supplementary Table S5. The taxonomic annotation data for the negative controls are shown in Additional file 2: Supplementary Table S6. Majority of the assigned reads in the negative controls were highly unlikely to be present in the human gut, suggesting no (or very little) impact of contamination on the archaeal 16S rRNA gene analysis.

\section{Estimation of archaeal abundance}

DNA was extracted from the faecal samples as described above. In total, 150 samples were randomly selected and subjected to real-time quantitative PCR [11] in three replicates. The archaeal primer sequences are listed in Additional file 2: Supplementary Table S3. Bacterial 16S rRNA gene (primers Bac1055YF and Bac1392R) was used as the control [63]. PCR was performed using the CFX96 $^{\text {TM }}$ real-time PCR detection system (Bio-Rad, Hercules, CA, USA) in a reaction volume of $20 \mu \mathrm{L}$ containing $10 \mu \mathrm{L}$ TOPreal qPCR 2X premix (Enzynomics, Daejeon, South Korea), $300 \mathrm{nM}$ of each of the forward and reverse primers and 2 ng template DNA. The Cq values were determined using the Bio-Rad CFX Manager software version 3.1. Escherichia coli $\mathrm{K} 12$ and Haloplanus salinus JCM $18368^{\mathrm{T}}$ were used to construct standard curves and perform quantitative analysis. The PCR efficiency and $R^{2}$ were $96.92 \%$ and 0.9995 for bacteria, and $97.57 \%$ and 0.9983 for archaea, respectively.

\section{Fluorescence in situ hybridisation analysis}

Fluorescence in situ hybridisation (FISH) analysis was performed according to a method by Hugenholtz [64] with minor modification. Briefly, the haloarchaeaspecific probe (HALO775) was designed using the ARB software [65]. The specificity of the HALO775 was evaluated based on the TestProbe in SILVA (SILVA SSU database v. 138) and the ProbeMatch in ribosomal database project (RDP, v. 11.5) databases (Additional file 2: Supplementary Tables S1 and S2). The designed probe, 
the bacterial 16S rRNA gene-targeting probe EUB338 [66] and non-specific probe NONEUB [67] were synthesised and labelled at the $5^{\prime}$ end with Cy3, Cy5 and FAM by Macrogen (Seoul, South Korea), respectively. To evaluate the possible cross-binding activity, we tested the binding activities of HALO775 to a haloarchaeon (Haloplanus salinus JCM $18368^{\mathrm{T}}$ ), bacterium (Escherichia coli $\mathrm{K} 12$ ) and another archaeon (Methanobrevibacter smithii JCM $30028^{\mathrm{T}}$ ). The cultured bacterium and the archaea were fixed with $4 \%$ paraformaldehyde in PBS at $4{ }^{\circ} \mathrm{C}$ for $4 \mathrm{~h}$, and the fixed samples were washed in PBS and gradually dehydrated in PBS-ethanol solution (final ratio of 1:1, vol/vol). Hybridisation was performed at $46{ }^{\circ} \mathrm{C}$ with $20 \%$ formamide hybridisation buffer. Afterwards, washing was performed again at $48{ }^{\circ} \mathrm{C}$. Both nonbinding probe (NONEUB $\mathrm{FAM}_{\mathrm{FA}}$ ) and no-probe controls were always included to decipher false positives. Samples were observed under a confocal microscope (LSM710; Carl Zeiss, Oberkochen, Germany) with $\times 1000$ magnification using the imaging software of ZEN v. 3.1 (blue edition, Carl Zeiss).

\section{Measurements of salinity and inorganic elements}

According to the relative abundance of haloarchaea, 20 faecal samples were selected. Each specimen $(200 \mathrm{mg})$ was diluted in $1 \mathrm{~mL}$ distilled water, and the salinity was measured using a salinity refractometer (Atago, Japan). The inorganic elements from these samples were measured using ICP-MS, as previously described [68].

\section{Statistical analysis}

Normality tests (Shapiro-Wilk) were carried out prior to correlation coefficient analysis and comparison of multiple samples. Correlation coefficient analysis was performed based on the relative abundance of the proportionally abundant archaeal taxa with respect to dietary nutrients, clinical metadata and food categories. Multiple samples were compared using the nonparametric Kruskal-Wallis test, followed by Dunn's multiple comparisons test. PERM ANOVA analysis was done based on the Bray-Curtis and Jaccard dissimilarity matrices, with 999 permutations. All statistical analyses were performed using the GraphPad Prism software v. $7.05\left({ }^{*} P<0.05,{ }^{* *} P<0.01\right.$ and ${ }^{* * *} P<$ $0.001)$.

\section{Supplementary information}

Supplementary information accompanies this paper at https://doi.org/10. 1186/s40168-020-00894-x.

Additional file 1. Supplementary Figures (S1-S7).

Additional file 2. Supplementary Tables (S1-S6).

\section{Abbreviations}

ASV: Amplicon sequence variant; DMSO: Dimethylsulphoxide;

FISH: Fluorescence in situ hybridisation; ICP-MS: Inductively coupled plasma- mass spectrometer; LDLC: Low-density lipoprotein cholesterol; PAM: Partitioning around medoids; PCoA: Principal coordinate analysis: PCR: Polymerase chain reaction; TotalC: Total cholesterol

\section{Acknowledgements}

The authors would like to thank Prof. Kwangwoo Kim at Kyung Hee University and Dr. Na-Ri Shin at Korea Research Institute of Bioscience and Biotechnology (KRIBB) for advising on the statistical analysis.

\section{Authors' contributions}

SWR and Y-DN conceived and designed the study; MYL and J-HK prepared the samples; JYK and YBK performed all the experiments; JYK, TWW, MYL, YBK, NK, M-SK, JK, SHL, W-HC, I-HN and H-JC analysed the data; SWR, JYK, TWW, NK, M-SK, H-JC and J-WB interpreted the data; JYK, MYL, YBK, W-HC and Y-DN performed the statistical and bioinformatics analyses; JYK, TWW and SWR wrote the manuscript. All authors provided helpful comments on the manuscript.

\section{Funding}

This research was supported by the Main Research Program of the Korea Food Research Institute (E0170601-03) and the World Institute of Kimchi (KE2001-2) funded by the Ministry of Science and ICT; the Basic Science Research Program of the National Research Foundation of Korea (2018R1D1A1A09082921).

\section{Availability of data and materials}

The sequencing reads have been deposited in the NCBI under the accession number PRJNA522626.

\section{Ethics approval and consent to participate}

The study protocol was approved by the Institutional Review Board (IRB) of Chung-Ang University Hospital (IRB File No. 1750-002-281) and was compliant with all relevant ethical regulations. Written informed consent was obtained from all participants.

\section{Consent for publication}

Written informed consent was obtained from all participants.

\section{Competing interests}

The authors declare that they have no competing interests.

\section{Author details}

${ }^{1}$ Microbiology and Functionality Research Group, World Institute of Kimchi, Gwangju 61755, Republic of Korea. ${ }^{2}$ Research Group of Healthcare, Research Division of Food Functionality, Korea Food Research Institute, Jeollabuk-do 55365, Republic of Korea. ${ }^{3}$ Geologic Environment Division, Korea Institute of Geoscience and Mineral Resources, Daejeon 34132, Republic of Korea.

${ }^{4}$ Department of Family Medicine, Chung-Ang University Hospital, Chung-Ang University College of Medicine, Seoul 06973, Republic of Korea. ${ }^{5}$ Department of Biology, Kyung Hee University, Seoul 02447, Republic of Korea.

Received: 12 March 2020 Accepted: 17 July 2020

Published online: 04 August 2020

\section{References}

1. Cani PD. Human gut microbiome: hopes, threats and promises. Gut. 2018; 67:1716-25

2. The Human Microbiome Project Consortium. A framework for human microbiome research. Nature. 2012;486:215-21.

3. Zhernakova A, Kurilshikov A, Bonder MJ, Tigchelaar EF, Schirmer M, Vatanen T, Mujagic Z, Vila AV, Falony G, Vieira-Silva S, et al. Population-based metagenomics analysis reveals markers for gut microbiome composition and diversity. Science. 2016;352:565-9.

4. Deschasaux M, Bouter KE, Prodan A, Levin E, Groen AK, Herrema H, Tremaroli V, Bakker GJ, Attaye I, Pinto-Sietsma SJ, et al. Depicting the composition of gut microbiota in a population with varied ethnic origins but shared geography. Nat Med. 2018:24:1526-31.

5. Duvallet C, Gibbons SM, Gurry T, Irizarry RA, Alm EJ. Meta-analysis of gut microbiome studies identifies disease-specific and shared responses. Nat Commun. 2017:8:1784. 
6. Karner MB, DeLong EF, Karl DM. Archaeal dominance in the mesopelagic zone of the Pacific Ocean. Nature. 2001;409:507-10.

7. Bintrim SB, Donohue TJ, Handelsman J, Roberts GP, Goodman RM. Molecular phylogeny of Archaea from soil. Proc Natl Acad Sci U S A. 1997; 94:277-82.

8. Caporaso JG, Lauber CL, Costello EK, Berg-Lyons D, Gonzalez A, Stombaugh J, Knights D, Gajer P, Ravel J, Fierer N, et al. Moving pictures of the human microbiome. Genome Biol. 2011;12:R50.

9. Kulik EM, Sandmeier H, Hinni K, Meyer J. Identification of archaeal rDNA from subgingival dental plaque by PCR amplification and sequence analysis. FEMS Microbiol Lett. 2001;196:129-33.

10. Miller TL, Wolin MJ. Enumeration of Methanobrevibacter smithii in human feces. Arch Microbiol. 1982:131:14-8.

11. Pausan MR, Csorba C, Singer G, Till H, Schopf V, Santigli E, Klug B, Hogenauer C, Blohs M, Moissl-Eichinger C. Exploring the Archaeome: Detection of Archaeal Signatures in the Human Body. Front Microbiol. 2019;10.

12. Nkamga VD, Henrissat B, Drancourt M. Archaea: Essential inhabitants of the human digestive microbiota. Hum Microbiome J. 2017;3:1-8.

13. Yim KJ, Kwon J, Cha IT, Oh KS, Song HS, Lee HW, Rhee JK, Song EJ, Rho JR Seo ML, et al. Occurrence of viable, red-pigmented haloarchaea in the plumage of captive flamingoes. Sci Rep. 2015;5:16425.

14. Roh SW, Kim KH, Nam YD, Chang HW, Park EJ, Bae JW. Investigation of archaeal and bacterial diversity in fermented seafood using barcoded pyrosequencing. ISME J. 2010;4:1-16.

15. Song HS, Cha IT, Rhee JK, Yim KJ, Kim AY, Choi JS, Baek SJ, Seo MJ, Park SJ, Nam YD, Roh SW. Halostella salina gen. nov., sp. nov., an extremely halophilic archaeon isolated from solar salt. Int J Syst Evol Microbiol. 2016; 66:2740-6.

16. Kim YB, Kim JY, Song HS, Lee C, Ahn SW, Lee SH, Jung MY, Rhee JK, Kim J, Hyun DW, et al. Novel haloarchaeon Natrinema thermophila having the highest growth temperature among haloarchaea with a large genome size. Sci Rep. 2018:8:7777.

17. Gaci N, Borrel G, Tottey W, O'Toole PW, Brugere JF. Archaea and the human gut: new beginning of an old story. World J Gastroenterol. 2014;20:16062-78.

18. Nam YD, Chang HW, Kim KH, Roh SW, Kim MS, Jung MJ, Lee SW, Kim JY, Yoon JH, Bae JW. Bacterial, archaeal, and eukaryal diversity in the intestines of Korean people. J Microbiol. 2008;46:491-501.

19. Seck EH, Senghor B, Merhej V, Bachar D, Cadoret F, Robert C, Azhar El, Yasir M, Bibi F, Jiman-Fatani AA, et al. Salt in stools is associated with obesity, gut halophilic microbiota and Akkermansia muciniphila depletion in humans. Int J Obes. 2019;43:862-71.

20. Khelaifia S, Raoult D. Haloferax massiliensis sp. nov., the first humanassociated halophilic archaea. New Microbes New Infect. 2016;12:96-8.

21. Almeida A, Nayfach S, Boland M, Strozzi F, Beracochea M, Shi ZJ, Pollard KS, Parks DH, Hugenholtz $P$, Segata $N$, et al. A unified sequence catalogue of over 280,000 genomes obtained from the human gut microbiome. bioRxiv. 2019.

22. Mahnert A, Blohs M, Pausan M-R, Moissl-Eichinger C. The human archaeome: methodological pitfalls and knowledge gaps. Emerg Top Life Sci. 2018;2:469.

23. Kim MS, Park EJ, Roh SW, Bae JW. Diversity and abundance of singlestranded DNA viruses in human feces. Appl Environ Microbiol. 2011;77: 8062-70.

24. Sender R, Fuchs S, Milo R. Revised estimates for the number of human and bacteria cells in the body. PLoS Biol. 2016;14:e1002533.

25. Stoddard SF, Smith BJ, Hein R, Roller BR, Schmidt TM. rrnDB: improved tools for interpreting rRNA gene abundance in bacteria and archaea and a new foundation for future development. Nucleic Acids Res. 2015; 43:D593-D598.

26. The Human Microbiome Project Consortium. Structure, function and diversity of the healthy human microbiome. Nature. 2012;486:207-14.

27. Arumugam M, Raes J, Pelletier E, Le Paslier D, Yamada T, Mende DR, Fernandes GR, Tap J, Bruls T, Batto JM, et al. Enterotypes of the human gut microbiome. Nature. 2011:473:174-80.

28. Koskinen K, Pausan MR, Perras AK, Beck M, Bang C, Mora M, Schilhabel A, Schmitz R, Moissl-Eichinger C. First insights into the diverse human archaeome: specific detection of archaea in the gastrointestinal tract, lung, and nose and on skin. mBio. 2017;8:e00824-17.

29. Moeller AH, Degnan PH, Pusey AE, Wilson ML, Hahn BH, Ochman H. Chimpanzees and humans harbour compositionally similar gut enterotypes. Nat Commun. 2012;3:1179.
30. Raymann K, Moeller AH, Goodman AL, Ochman H. Unexplored archaeal diversity in the great ape gut microbiome. mSphere. 2017;2.

31. Florin TH, Zhu G, Kirk KM, Martin NG. Shared and unique environmental factors determine the ecology of methanogens in humans and rats. Am J Gastroenterol. 2000;95:2872-9.

32. Eckburg PB, Bik EM, Bernstein CN, Purdom E, Dethlefsen L, Sargent M, Gill $\mathrm{SR}$, Nelson KE, Relman DA. Diversity of the human intestinal microbial flora. Science. 2005;308:1635-8.

33. Lepp PW, Brinig MM, Ouverney CC, Palm K, Armitage GC, Relman DA. Methanogenic archaea and human periodontal disease. Proc Natl Acad Sci U S A. 2004;101:6176-81.

34. Eckburg PB, Lepp PW, Relman DA. Archaea and their potential role in human disease. Infect Immun. 2003;71:591-6.

35. Chaudhary PP, Conway PL, Schlundt J. Methanogens in humans: potentially beneficial or harmful for health. Appl Microbiol Biotechnol. 2018;102:3095-104.

36. Oxley AP, Lanfranconi MP, Wurdemann D, Ott S, Schreiber S, McGenity TJ, Timmis KN, Nogales B. Halophilic archaea in the human intestinal mucosa. Environ Microbiol. 2010;12:2398-410.

37. Oren A: Haloplanus. In Bergey's manual of systematics of archaea and bacteria. Edited by W. B. Whitman FR, P. Kämpfer, M. Trujillo, J. Chun, P. DeVos, B. Hedlund and S. Dedysh; 2016. p. 1-10.

38. Oren A: Halorubrum. In Bergey's manual of systematics of archaea and bacteria. Edited by W. B. Whitman FR, P. Kämpfer, M. Trujillo, J. Chun, P. DeVos, B. Hedlund and S. Dedysh; 2018. p. 1-48.

39. Lee HS. Diversity of halophilic archaea in fermented foods and human intestines and their application. J Microbiol Biotechnol. 2013;23:1645-53.

40. Tamang JP, Watanabe K, Holzapfel WH. Review: diversity of microorganisms in global fermented foods and beverages. Front Microbiol. 2016;7:377.

41. Purdy KJ, Cresswell-Maynard TD, Nedwell DB, McGenity TJ, Grant WD, Timmis KN, Embley TM. Isolation of haloarchaea that grow at low salinities. Environ Microbiol. 2004:6:591-5.

42. Fukushima T, Usami R, Kamekura M. A traditional Japanese-style salt field is a niche for haloarchaeal strains that can survive in $0.5 \%$ salt solution. Saline Systems. 2007;3:2

43. Andrei AS, Banciu HL, Oren A. Living with salt: metabolic and phylogenetic diversity of archaea inhabiting saline ecosystems. FEMS Microbiol Lett. 2012; 330:1-9.

44. Cani PD. Gut cell metabolism shapes the microbiome. Science. 2017;357: 548-9.

45. Rivera-Chavez F, Lopez CA, Baumler AJ. Oxygen as a driver of gut dysbiosis. Free Radic Biol Med. 2017;105:93-101.

46. Sorokin DY, Kublanov IV, Gavrilov SN, Rojo D, Roman P, Golyshin PN, Slepak VZ, Smedile F, Ferrer M, Messina E, et al. Elemental sulfur and acetate can support life of a novel strictly anaerobic haloarchaeon. ISME J. 2016;10:240-52.

47. Sorokin DY, Messina E, Smedile F, Roman P, Damste JSS, Ciordia S, Mena MC, Ferrer M, Golyshin PN, Kublanov IV, et al. Discovery of anaerobic lithoheterotrophic haloarchaea, ubiquitous in hypersaline habitats. ISME J. 2017;11:1245-60

48. Jeffery IB, Claesson MJ, O'Toole PW, Shanahan F. Categorization of the gut microbiota: enterotypes or gradients? Nat Rev Microbiol. 2012;10:591-2.

49. Ahn Y, Kwon E, Shim JE, Park MK, Joo Y, Kimm K, Park C, Kim DH. Validation and reproducibility of food frequency questionnaire for Korean genome epidemiologic study. Eur J Clin Nutr. 2007;61:1435-41.

50. Lim MY, Nam YD. Population-based gut microbiome associations with intrinsic and extrinsic host factors and serum metabolome in Koreans. Submitted for publication.

51. Lim MY, Song EJ, Kim SH, Lee J, Nam YD. Comparison of DNA extraction methods for human gut microbial community profiling. Syst Appl Microbiol. 2018;41:151-7.

52. Bolyen E, Rideout JR, Dillon MR, Bokulich NA, Abnet CC, Al-Ghalith GA, Alexander H, Alm EJ, Arumugam M, Asnicar F, et al. Reproducible, interactive, scalable and extensible microbiome data science using QIIME 2. Nat Biotechnol. 2019;37:852-7.

53. Callahan BJ, McMurdie PJ, Rosen MJ, Han AW, Johnson AJ, Holmes SP. DADA2: High-resolution sample inference from Illumina amplicon data. Nat Methods. 2016:13:581-3.

54. Pedregosa F, Varoquaux G, Gramfort A, Michel V, Thirion B, Grisel O, Blondel $M$, Prettenhofer $P$, Weiss R, Dubourg $V$, et al. Scikit-learn: Machine learning in Python. J Mach Learn Res. 2011;12:2825-30. 
55. Quast C, Pruesse E, Yilmaz P, Gerken J, Schweer T, Yarza P, Peplies J, Glockner FO. The SILVA ribosomal RNA gene database project: improved data processing and web-based tools. Nucleic Acids Res. 2013;41:D590-6.

56. Kumar S, Stecher G, Li M, Knyaz C, Tamura K. MEGA X: Molecular. evolutionary genetics analysis across computing platforms. Mol Biol Evol. 2018;35:1547-1549.

57. Letunic I, Bork P. Interactive tree of life (iTOL) V3: an online tool for the display and annotation of phylogenetic and other trees. Nucleic Acids Res. 2016:44:W242-5.

58. Lim MY, Rho M, Song YM, Lee K, Sung J, Ko G. Stability of gut enterotypes in Korean monozygotic twins and their association with biomarkers and diet. Sci Rep. 2014;4:7348.

59. Kaufman L, Rousseeuw PJ: Partitioning around medoids (Program PAM). In finding groups in data: an introduction to cluster analysis. Hoboken, NJ: John Wiley and Sons, Inc: 1990. p. 68-125.

60. Bray JR, Curtis JT. An ordination of the upland forest communities of southern Wisconsin. Ecological Monographs. 1957;27:325-49.

61. Rousseeuw PJ. Silhouettes: A graphical aid to the interpretation and validation of cluster analysis. J Comput Appl Math. 1987;20:53-65.

62. Mitchell AL, Almeida A, Beracochea M, Boland M, Burgin J, Cochrane G, Crusoe MR, Kale V, Potter SC, Richardson LJ, et al. MGnify: the microbiome analysis resource in 2020. Nucleic Acids Res. 2020:48:D570-D8.

63. Ritalahti KM, Amos BK, Sung Y, Wu Q, Koenigsberg SS, Loffler FE. Quantitative PCR targeting 165 rRNA and reductive dehalogenase genes simultaneously monitors multiple Dehalococcoides strains. Appl Environ Microbiol. 2006;72:2765-74.

64. Hugenholtz P, Tyson GW, Blackall LL: Design and evaluation of 165 rRNAtargeted oligonucleotide probes for fluorescence in situ hybridization. In Gene probes: Principles and protocols. Edited by de Muro MA, Rapley R. Totowa, NJ: Humana Press; 2002. p. 29-42.

65. Ludwig W, Strunk O, Westram R, Richter L, Meier H. Yadhukumar, Buchner A, Lai T, Steppi S, Jobb G, et al. ARB: a software environment for sequence data. Nucleic Acids Res. 2004;32:1363-71.

66. Amann RI, Binder BJ, Olson RJ, Chisholm SW, Devereux R, Stahl DA. Combination of $16 \mathrm{~S}$ rRNA-targeted oligonucleotide probes with flow cytometry for analyzing mixed microbial populations. Appl Environ Microbiol. 1990:56:1919-25.

67. Wallner G, Amann R, Beisker W. Optimizing fluorescent in situ hybridization with rRNA-targeted oligonucleotide probes for flow cytometric identification of microorganisms. Cytometry. 1993;14:136-43.

68. Lee HW, Lim NL, Cho K, Yang HY, Yim KJ, Kim MJ, Lee M, Kim DH, Koh HB, Jung WK, et al. Characterisation of inorganic elements and volatile organic compounds in the dried sea cucumber Stichopus japonicus. Food Chem. 2014;147:34-41.

\section{Publisher's Note}

Springer Nature remains neutral with regard to jurisdictional claims in published maps and institutional affiliations.

Ready to submit your research? Choose BMC and benefit from:

- fast, convenient online submission

- thorough peer review by experienced researchers in your field

- rapid publication on acceptance

- support for research data, including large and complex data types

- gold Open Access which fosters wider collaboration and increased citations

- maximum visibility for your research: over $100 \mathrm{M}$ website views per year

At $\mathrm{BMC}$, research is always in progress.

Learn more biomedcentral.com/submissions 